\title{
Effect of a fat spread enriched with medium-chain triacylglycerols and a special fatty acid-micronutrient combination on cardiometabolic risk factors in overweight patients with diabetes
}

\author{
Roswitha Siener ${ }^{1}$, Christina Ehrhardt ${ }^{2}$, Norman Bitterlich ${ }^{3}$ and Christine Metzner ${ }^{2,4^{*}}$
}

\begin{abstract}
Background: Medium-chain triacylglycerols (MCT), omega-3 polyunsaturated fatty acids (n-3-PUFA) and micronutrients may be useful for weight and cardiometabolic risk management. However, studies analyzing the effect of a combination of both in individuals at increased cardiometabolic risk are lacking. Therefore, this randomized, controlled, double-blind study investigated the effect of a fat spread enriched with two different doses of MCT and a special long-chain fatty acid-micronutrient combination on cardiometabolic risk factors in overweight diabetic patients.
\end{abstract}

Methods: Fifty-four patients received either a fat spread with $6 \mathrm{~g} / \mathrm{d} \mathrm{MCT}$ (MCT30\%) or $1.2 \mathrm{~g} / \mathrm{d}$ (MCT6\%). Forty-three completed the study. Analysis was performed according to the median of MCT intake (supplemented and foodderived MCT). Clinical, anthropometric, blood, 24 h-urine parameters and dietary intake were assessed at baseline and after 12 weeks.

Results: Total MCT intake $>7 \mathrm{~g} / \mathrm{d}$ (MCT $>7$ group) significantly reduced waist circumference (WC) by $1.81 \pm 2.69$ $\mathrm{cm}$, whereas $\leq 7 \mathrm{~g} / \mathrm{d}$ MCT (MCT $\leq 7$ group) increased WC by $0.32 \pm 3.03 \mathrm{~cm}(p=0.027)$, which was supported by a change in waist-to-height ratio (WHtR) $(p=0.018)$. Fasting serum triglycerides (TG) increased in both groups over time due to dietary habits. In contrast, diabetic metabolic situation and urinary albumin excretion did not alter. Urinary $\mathrm{pH}$ differed significantly between groups after 12 weeks.

Conclusion: An intake of $>7 \mathrm{~g} / \mathrm{d}$ MCT reduced WC in overweight diabetics, whereas the increase in the intake of fatty acids may have worsened fasting TG. Therefore, the suitability of a fat for nutrient enrichment remains to be challenged, and further studies in low-fat matrices are desirable.

\section{Introduction}

Evidence indicates the importance of abdominal adipose tissue as endocrine tissue being key for cardiometabolic risk factors [1]. Among patients with type 2 diabetes the combination of abdominal enlargement and hypertriglyceridemia, so-called hypertriglyceridemic waist (HW), is highly prevalent [2] and has been associated with a greater degree of subclinical atherosclerosis that may be related to the

\footnotetext{
* Correspondence: christine.metzner@rwth-aachen.de

${ }^{2}$ Bonn Education Association for Dietetics r. A., Cologne, Germany

Full list of author information is available at the end of the article
}

proatherogenic lipoprotein changes [3,4]. These lipoprotein changes called atherogenic dyslipidemia are typically characterized by reduced concentrations of HDL cholesterol (HDL-C), elevated triglycerides (TG), and elevated small, dense LDL particles [5]. HW has also been proposed to be an important factor increasing $\mathrm{C}$-reactive protein (CRP) concentrations and relative coronary risk in patients with type 2 diabetes of any age and sex [6,7]. Moreover, reactive oxygen species are produced in various tissues under diabetic conditions leading to an antioxidant depletion and

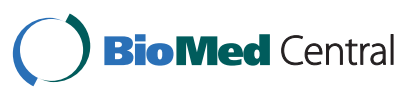

(c) 2011 Siener et al; licensee BioMed Central Ltd. This is an Open Access article distributed under the terms of the Creative Commons Attribution License (http://creativecommons.org/licenses/by/2.0), which permits unrestricted use, distribution, and reproduction in any medium, provided the original work is properly cited. 
increased lipid oxidation, advanced glycation end products (AGE), cell damage and endothelial dysfunction $[8,9]$.

A supplementation of medium-chain triacylglycerols (MCT) for conventional dietary fats has been proposed as beneficial for weight management, since MCT are rapidly absorbed and preferentially transported through the portal venous system to the liver. The subsequent stimulation of $\beta$-oxidation in hepatocytes may reduce the circulating fatty acids available to the adipocytes [10]. Moreover, MCT enhances energy expenditure following thermogenesis [11-13].

The cardiometabolic protective effects of omega-3 polyunsaturated fatty acids ( $n$-3-PUFA) appear to be due to a synergism between multiple mechanisms. These involve anti-inflammatory, inflammation-resolving, anti-arrhythmic and anti-thrombotic effects as well as a regulation of transcription factors, gene expression and membrane fluidity [14]. Moreover, n-3-PUFA, in particular eicosapentaenoic acid (EPA) and docosahexaenoic acid (DHA), have been demonstrated to exert beneficial effects in lowering TG concentrations [15], including in patients with atherogenic dyslipidemia associated diabetes [16,17].

Since the fat spreads were also enriched with vitamins and minerals, their consumption may add to an increase in the daily supply of these essential micronutrients, and thus ameliorate nutritional status. These changes may exert favourable effects on weight management and cardiometabolic risk factors $[18,19]$.

As fat spread is a central source of fat in the diet, the choice of a spread providing a high-quality fat and micronutrient profile may be an important dietary measure for patients with diabetes. Fat spreads characterized by a combination of MCT, n-3-PUFA and micronutrients have not been evaluated so far in this cohort. Therefore, aim of this study was to assess the benefit of a fat spread characterized by the above mentioned combination on cardiometabolic risk factors in overweight diabetic patients.

\section{Methods}

\section{Participants}

Overweight diabetic patients were recruited from the region of Altomuenster, Bavaria, Germany. Both men and women aged 30 to 82 years with a body mass index (BMI) of $27 \mathrm{~kg} / \mathrm{m}^{2}$ or greater and a waist circumference (WC) $\geq 94 \mathrm{~cm}$ for women and $\geq 102 \mathrm{~cm}$ for men were included into the study. Overweight diabetic patients were defined by clinical criteria. Individuals taking any medication or nutritional supplements for weight reduction were excluded. Further exclusion criteria comprised the supplementation and/or therapy with marine n-3PUFA, micronutrients, treatment with glitazones and/or telmisartan, insulin-dependent diabetes, ketoacidosis and acute or chronic diarrhea. The study was approved by the Ethics Committee of the Bavarian Chamber of Physicians, Munich, Germany, and all patients provided informed consent before study onset.

\section{Study design}

This prospective study was carried out in a double-blind controlled manner. Patients were randomly assigned to receive a fat spread with either $6 \mathrm{~g} / \mathrm{d}$ (MCT30\%) or $1.2 \mathrm{~g} / \mathrm{d}$ MCT (MCT6\%), which were equally enriched with special unsaturated fatty acids and micronutrients (Table 1). Patients were asked to consume $2 \times 15 \mathrm{~g} / \mathrm{d}$ of the fat spreads (MCT30\% or MCT6\%, respectively) for 12 weeks as substitution for their usual dietary spread

Table 1 Mean composition of the MCT30\% and MCT6\% fat spread per daily serving $(2 \times 15 \mathrm{~g})^{1}$

\begin{tabular}{lcc}
\hline & MCT30\% & MCT6\% \\
\hline Energy (kcal) & 176 & 176 \\
Carbohydrates (g) & 0 & 0 \\
Protein (g) & 0 & 0 \\
Fat (g) & 19.5 & 19.5
\end{tabular}

Fatty acids

$\begin{array}{lll}\text { SAFA (g) } & 8.7 & 7.2 \\ \text { - MCT (g) } & 6.0 & 1.2 \\ \text { - SAFA without MCT (g) } & 2.7 & 6.0 \\ \text { MUFA (g) } & 6.9 & 8.1 \\ \text { - Oleic acid (g) } & 6.6 & 7.8 \\ \text { PUFA (g) } & 3.9 & 4.2 \\ \text { - LA (g) } & 2.88 & 3.1 \\ \text { - ALA (g) } & 0.87 & 0.9 \\ \text { - EPA (g) } & 0.15 & 0.15 \\ \text { - DHA (g) } & 0.09 & 0.09 \\ \text { Trans fatty acids (g) } & 0.12 & 0.09 \\ \text { lesterol (g) } & 0.006 & 0.006\end{array}$

Micronutrients

$\begin{array}{lcc}\text { Vitamin A }(\mu \mathrm{g} \mathrm{RE}) & 240 & 240 \\ \text { Vitamin B1 }(\mathrm{mg}) & 0.45 & 0.45 \\ \text { Vitamin B2 }(\mathrm{mg}) & 0.48 & 0.48 \\ \text { Nicotinic acid }(\mathrm{mg}) & 4.95 & 4.95 \\ \text { Vitamin B6 }(\mathrm{mg}) & 0.48 & 0.48 \\ \text { Folic acid }(\mu \mathrm{g}) & 120 & 120 \\ \text { Vitamin B12 }(\mu \mathrm{g}) & 1.95 & 1.95 \\ \text { Vitamin D3 }(\mu \mathrm{g}) & 1.8 & 1.8 \\ \text { Vitamin E }(\mathrm{mg}) & 3.3 & 3.3 \\ \text { Sodium }(\mathrm{g}) & 0.002 & 0.002 \\ \text { Chrome }(\mathrm{mg}) & 0.015 & 0.015 \\ \text { Manganese }(\mathrm{mg}) & 0.6 & 0.6\end{array}$

1 abbreviations used: ALA, alpha-linolenic acid; EPA, eicosapentaenoic acid; DHA, docosahexaenoic acid; LA, linolenic acid; MCT, medium-chain triacylglycerols; MUFA, monounsaturated fatty acids; PUFA, polyunsaturated fatty acids; RE, retinol equivalent; SAFA, saturated fatty acids. 
and to maintain their common diet and physical activity level during the study.

Data were analyzed at baseline and 12 weeks of intervention. Body weight $(\mathrm{kg})$, height $(\mathrm{cm})$ and $W C(\mathrm{~cm})$ were measured. WC was determined to the nearest 0.1 via flexible tape and measurement was documented by photography. Anthropometric measurements and venous blood samples were performed in the morning after an overnight fasting period of at least $12 \mathrm{~h}$. Dietary intake data (3-day food record) was analyzed using PRODI 5.5 software (WVG, Stuttgart, Germany). The average of three days was assessed. Glomerular filtration rate (GFR) was calculated using the Modification of Diet in Renal Disease (MDRD) study equation [20]. Compliance was monitored by weighing of returned spread tubs at week 8 and 12 of intervention as well as by evaluating dietary records on which the number of spread servings per day were recorded. Analysis was performed according to the median of MCT intake (supplemented and food-derived MCT), which was $7 \mathrm{~g} / \mathrm{d}$ MCT.

\section{Laboratory methods}

SYSCOMP GmbH, Augsburg, Germany, conducted all laboratory analyses. Analysis of serum glucose (hexokinase method), uric acid (uricase-PAP method), gammaglutamyltransferase (GGT), aspartate-aminotransferase (ASAT), alanine-aminotransferase (ALAT) (IFCC method), glycated haemoglobin (HbA1c) (turbidimetric immunologic inhibition assay (TINIA)), insulin (ECLIA), total cholesterol (TC) (CHOD-PAP method), LDL-C and HDL-C (enzymatic colour test), TG (GPO-PAP method), CRP sensitive (turbidimetry) and 24 h urinary albumin (turbidimetry), $\mathrm{pH}$ (Combur test) and sodium (ion sensitive electrode (ISE)) were measured on Roche analyser.

\section{Statistical methods}

All data are presented as means \pm standard deviation. Significance was set at p-value 0.05 (two-sided). Changes of WC and body weight from baseline were determined using non-parametric analyses (exact Mann-Whitney-Utest). Pre-post intervention changes of all other variables were analyzed via non-parametric Wilcoxon test and inter-group differences were compared using MannWhitney-U test. ANCOVA including use of WC change as a covariate was used to control for the potential confounder of serum fasting TG. Data analysis was performed based on the per protocol population and by using SPSS $^{\circledR}$ for Windows (version PASW 18.0).

\section{Results}

\section{Participants}

A total of 54 overweight diabetic patients were recruited, of whom 43 were included into the per protocol (PP) population (23 men and 20 women). Reasons for an exclusion from the PP population included poor compliance as determined by more than $20 \%$ variance of product consumption $(n=7)$ and variation in study duration of more than 7 days $(n=2)$. Moreover, 2 patients left the study due to personal reasons (additional file 1). Both fat spreads were well tolerated by the patients and the majority of patients followed the treatment without any reported difficulty.

Male and female subjects were equally distributed (12 and 9 in the MCT > 7 group, 11 and 11 in the MCT $\leq$ 7 group, respectively; $\mathrm{p}=0.639$ ). Metformin was used as anti-diabetic medication by 20 and sulfonylurea by 13 patients. Statins were taken as cholesterol-lowering medication by 11 and antihypertensive medication (beta-blocker, angiotensin converting enzyme inhibitors and calcium channel blocker) by 49 patients.

\section{Clinical measurements}

A total MCT intake $>7 \mathrm{~g} / \mathrm{d}(\mathrm{n}=21)$ significantly reduced $\mathrm{WC}$ by $1.81 \pm 2.69 \mathrm{~cm}(\mathrm{p}=0.005)$, whereas no significant change was observed in the $\mathrm{MCT} \leq 7$ group $(\mathrm{n}=22)(0.32 \pm 3.03 \mathrm{~cm}, \mathrm{p}=1.000)$ over time (Table $2)$. The inter-group changes differed significantly ( $\mathrm{p}=$ 0.027). Additionally, a significant difference in waist-toheight ratio (WHtR) was observed between groups ( $\mathrm{p}=$ 0.018), whereas BMI and body weight changes did not change significantly. Systolic and diastolic blood pressure remained unaffected by the supplementation in both groups.

\section{Serum parameters}

The average value of fasting TG was below the cut-off level of $150 \mathrm{mg} / \mathrm{dL}$ in the MCT > 7 group $(147.3 \pm 92.0 \mathrm{mg} / \mathrm{dL})$, whereas the fasting triglyceride level of the MCT $\leq 7$ group was hypertriglyceridemic $(166.5 \pm 113.3 \mathrm{mg} / \mathrm{dL})$ (Table 2$)$. Over time, fasting TG increased significantly in both groups $(\mathrm{p}=0.021$ and $\mathrm{p}=0.022$, respectively), but there was no inter-group difference. Total cholesterol (TC), LDL-C, HDL-C, non-HDL-C and TC/HDL-C ratio did not change during supplementation. Fasting glucose, insulin, HOMA index, HbA1c and CRP were elevated in both groups during the study, but were not affected by intervention. The same applies to ASAT and ALAT, however GGT increased significantly in the MCT $\leq 7$ group ( $p=0.023)$. In the $\mathrm{MCT}>7$ group, uric acid increased significantly during supplementation $(\mathrm{p}=$ 0.024), but remained within the normal range.

\section{Urine parameters}

Whereas no changes were observed for GFR, urinary albumin and sodium excretion, urinary $\mathrm{pH}$ varied significantly between groups after 12 weeks of intervention $(\mathrm{p}=0.032)$ (Table 2$)$. 
Table 2 Clinical and biochemical characteristics at baseline and after supplementation ${ }^{1,2}$

\begin{tabular}{|c|c|c|c|c|c|c|c|c|c|}
\hline & \multicolumn{4}{|c|}{ MCT intake $>7 \mathrm{~g} / \mathrm{d}(\mathrm{n}=21)$} & \multicolumn{4}{|c|}{ MCT intake $\leq 7 \mathrm{~g} / \mathrm{d}(\mathrm{n}=22)$} & \multirow[t]{2}{*}{$P^{b}$} \\
\hline & wk 0 & wk 12 & wk $12-0$ & $P^{a}$ & wk 0 & wk12 & wk $12-0$ & $\mathrm{P}^{\mathrm{a}}$ & \\
\hline Age (years) & $67.3 \pm 9.3$ & & & & $66.3 \pm 7.2$ & & & & 0.512 \\
\hline Weight (kg) & $95.7 \pm 21.2$ & $95.3 \pm 21.7$ & $-0.35 \pm 1.92$ & 0.641 & $87.7 \pm 11.6$ & $88.3 \pm 11.7$ & $0.54 \pm 1.76$ & 0.118 & 0.177 \\
\hline BMI $\left(\mathrm{kg} / \mathrm{m}^{2}\right)$ & $34.35 \pm 6.84$ & $34.23 \pm 7.08$ & $-0.12 \pm 0.70$ & 0.681 & $31.69 \pm 4.05$ & $31.89 \pm 4.10$ & $0.20 \pm 0.61$ & 0.092 & 0.174 \\
\hline$W C(\mathrm{~cm})$ & $112.5 \pm 12.5$ & $110.7 \pm 12.9$ & $-1.81 \pm 2.69$ & 0.005 & $106.4 \pm 6.6$ & $106.7 \pm 7.0$ & $0.32 \pm 3.03$ & 1.000 & 0.027 \\
\hline $\mathrm{WHtR}$ & $0.676 \pm 0.076$ & $0.665 \pm 0.079$ & $-0.010 \pm 0.016$ & 0.008 & $0.640 \pm 0.045$ & $0.642 \pm 0.049$ & $0.002 \pm 0.018$ & 0.920 & 0.018 \\
\hline SBP $(\mathrm{mmHg})$ & $138.6 \pm 18.4$ & $137.9 \pm 12.0$ & $-0.7 \pm 13.2$ & 0.792 & $148.4 \pm 18.6$ & $142.0 \pm 17.2$ & $-6.4 \pm 15.4$ & 0.069 & 0.249 \\
\hline $\mathrm{DBP}(\mathrm{mmHg})$ & $84.8 \pm 11.7$ & $84.1 \pm 7.7$ & $-0.7 \pm 10.3$ & 0.707 & $87.7 \pm 8.7$ & $85.2 \pm 8.7$ & $-2.5 \pm 9.2$ & 0.170 & 0.612 \\
\hline Fasting TG (mg/dl) & $147.3 \pm 92.0$ & $181.6 \pm 122.7$ & $34.2 \pm 72.6$ & 0.021 & $166.5 \pm 113.3$ & $216.6 \pm 177.1$ & $50.1 \pm 108.1$ & 0.022 & 0.990 \\
\hline $\mathrm{TC}(\mathrm{mg} / \mathrm{dl})$ & $209.5 \pm 41.0$ & $214.0 \pm 41.6$ & $4.52 \pm 18.60$ & 0.177 & $217.7 \pm 37.9$ & $225.4 \pm 42.7$ & $7.68 \pm 23.04$ & 0.058 & 0.527 \\
\hline LDL-C (mg/dl) & $128.8 \pm 34.9$ & $130.7 \pm 32.3$ & $1.95 \pm 16.59$ & 0.357 & $136.4 \pm 34.9$ & $135.6 \pm 40.1$ & $-0.86 \pm 22.04$ & 0.848 & 0.618 \\
\hline $\mathrm{HDL}-\mathrm{C}(\mathrm{mg} / \mathrm{dl})$ & $52.1 \pm 10.8$ & $49.7 \pm 10.6$ & $-2.39 \pm 8.75$ & 0.230 & $49.5 \pm 9.9$ & $51.4 \pm 13.9$ & $1.88 \pm 6.71$ & 0.363 & 0.085 \\
\hline TC/HDL-C & $4.15 \pm 0.98$ & $4.50 \pm 1.37$ & $0.35 \pm 0.82$ & 0.064 & $4.51 \pm 1.01$ & $4.65 \pm 1.50$ & $0.14 \pm 0.70$ & 0.637 & 0.289 \\
\hline Non-HDL-C (mg/dl) & $157.4 \pm 40.3$ & $164.3 \pm 43.5$ & $6.91 \pm 18.8$ & 0.058 & $168.2 \pm 35.6$ & $174.0 \pm 42.8$ & $5.80 \pm 22.7$ & 0.158 & 0.981 \\
\hline Fasting glucose (mg/dl) & $127.5 \pm 21.8$ & $132.0 \pm 29.7$ & $4.48 \pm 19.91$ & 0.422 & $124.4 \pm 23.9$ & $126.5 \pm 34.6$ & $2.09 \pm 18.70$ & 0.676 & 0.313 \\
\hline Fasting insulin $(\mu \mathrm{U} / \mathrm{ml})$ & $12.6 \pm 9.1$ & $13.7 \pm 11.0$ & $1.09 \pm 9.33$ & 0.509 & $14.4 \pm 6.8$ & $17.6 \pm 25.4$ & $3.17 \pm 24.16$ & 0.299 & 0.177 \\
\hline HOMA-Index & $4.14 \pm 3.73$ & $4.81 \pm 5.52$ & $0.68 \pm 5.21$ & 0.639 & $4.46 \pm 2.39$ & $5.72 \pm 9.01$ & $1.26 \pm 8.68$ & 0.306 & 0.234 \\
\hline HbA1c (\%) & $6.76 \pm 0.58$ & $6.69 \pm 0.78$ & $-0.067 \pm 0.380$ & 0.367 & $6.46 \pm 0.47$ & $6.46 \pm 0.53$ & $0.004 \pm 0.208$ & 0.844 & 0.179 \\
\hline CRP (mg/l) & $6.17 \pm 5.97$ & $5.52 \pm 4.91$ & $-0.66 \pm 2.77$ & 0.140 & $5.80 \pm 8.46$ & $4.45 \pm 4.63$ & $-1.35 \pm 6.76$ & 0.274 & 0.961 \\
\hline Uric acid (mg/l) & $5.96 \pm 1.53$ & $6.27 \pm 1.52$ & $0.31 \pm 0.66$ & 0.024 & $6.58 \pm 1.26$ & $6.58 \pm 1.04$ & $0.00 \pm 1.09$ & 0.944 & 0.103 \\
\hline GGT (U/l) & $43.8 \pm 50.1$ & $41.2 \pm 26.4$ & $-2.57 \pm 27.84$ & 0.265 & $43.8 \pm 47.6$ & $44.2 \pm 30.5$ & $0.45 \pm 20.82$ & 0.023 & 0.442 \\
\hline ASAT (U/I) & $28.4 \pm 10.6$ & $28.0 \pm 8.3$ & $-0.43 \pm 5.97$ & 0.861 & $28.1 \pm 11.9$ & $27.1 \pm 6.5$ & $-1.00 \pm 9.01$ & 0.480 & 0.643 \\
\hline ALAT (U/I) & $33.5 \pm 21.8$ & $31.2 \pm 14.3$ & $-2.24 \pm 9.96$ & 0.650 & $29.2 \pm 14.1$ & $30.4 \pm 13.8$ & $1.18 \pm 10.77$ & 0.166 & 0.607 \\
\hline GFR $\left(\mathrm{mL} / \mathrm{min} / 1.73 \mathrm{~m}^{2}\right)$ & $87.9 \pm 19.1$ & $86.4 \pm 22.4$ & $-1.54 \pm 11.28$ & 0.433 & $81.7 \pm 15.7$ & $83.4 \pm 14.9$ & $1.71 \pm 8.60$ & 0.095 & 0.290 \\
\hline U-Albumin (mg/24 h) & $55.3 \pm 199.4$ & $19.5 \pm 39.9$ & $-35.8 \pm 162.7$ & 0.594 & $62.1 \pm 225.8$ & $60.0 \pm 150.1$ & $-2.1 \pm 109.3$ & 0.641 & 0.842 \\
\hline U-pH (24 h) & $5.81 \pm 0.81$ & $5.52 \pm 0.73$ & $-0.29 \pm 0.70$ & 0.062 & $5.80 \pm 1.08$ & $6.05 \pm 1.12$ & $0.25 \pm 0.88$ & 0.176 & 0.032 \\
\hline U-Sodium (mmol/24 h) & $212.0 \pm 84.6$ & $234.5 \pm 89.0$ & $22.5 \pm 78.2$ & 0.145 & $208.6 \pm 67.6$ & $231.0 \pm 75.6$ & $22.4 \pm 72.2$ & 0.153 & 1.000 \\
\hline
\end{tabular}

${ }^{1}$ data are presented as mean \pm SD.

${ }^{a} \mathrm{p}$ values for comparison between week 0 and 12 (Wilcoxon-Test).

${ }^{b} \mathrm{p}$ value for comparison of group $\times$ time interactions (Mann-Whitney-U-Test).

2 abbreviations used: ASAT, aspartate-aminotransferase; ALAT, alanine-aminotransferase; CRP, C reactive protein; DBP, diastolic blood pressure; GFR, glomerular filtration rate; GGT, gamma-glutamyltransferase; HbA1c, glycated haemoglobin; HDL-C, HDL cholesterol; LDL-C, LDL cholesterol; MCT, medium-chain triacylglycerols; SBP, systolic blood pressure; TC, total cholesterol; TG, triglycerides; WC, waist circumference; WHtR, waist to height ratio; U-albumin, urinary albumin; U-pH, urinary $\mathrm{pH}$; U-sodium, urinary sodium.

\section{Dietary intake}

Except for the MCT intake, there was no significant difference in macro- and micronutrient consumption between both groups (Table 3). However, the relation of macronutrients shifted due to changes in dietary habits. Fat intake and percentage of energy consumed from fat increased during supplementation, however this change was not significant in the MCT $>7$ group. Saturated fat intake was found to be significantly elevated in the $\mathrm{MCT}>7$ group by $7.3 \pm 11.7 \mathrm{~g} / \mathrm{d}(\mathrm{p}=0.016)$, mainly due to supplementation. PUFA consumption increased significantly only in the MCT > 7 group $(p=0.033)$, whereas monounsaturated fatty acid (MUFA) intake, including oleic acid, was significantly higher following fat spread consumption in both groups ( $\mathrm{p}<0.039$ and 0.010 , respectively). The same applied for daily alphalinolenic acid (ALA), EPA and DHA intake. Glucose, fructose, sorbitol and purine intake remained stable over time. However, the fat spread consumption affected daily vitamin supply, as revealed by a significant increase in vitamin D, B2 and folic acid intake after 12 weeks in both groups. Vitamin E intake rose in the MCT > 7 group, and nicotinic acid as well as vitamin B6 consumption were significantly enhanced over time in the MCT $\leq 7$ group, without significant differences between groups.

\section{Discussion}

To our knowledge this is the first study investigating the effect of a fat spread enriched with MCT and a special fatty acid-micronutrient combination on cardiometabolic risk factors in overweight diabetic patients. The major finding of the present study was a significant decrease in $\mathrm{WC}$ in the $\mathrm{MCT}>7$ group. The reduction in $\mathrm{WC}$ 
Table 3 Daily dietary intake at baseline and after supplementation ${ }^{1,2}$

\begin{tabular}{|c|c|c|c|c|c|c|c|c|c|}
\hline & \multicolumn{4}{|c|}{ MCT intake > $7 \mathrm{~g} / \mathrm{d}(\mathrm{n}=21)$} & \multicolumn{4}{|c|}{ MTC intake $\leq 7 \mathrm{~g} / \mathrm{d}(\mathrm{n}=22)$} & \multirow[t]{2}{*}{$\mathrm{P}^{\mathbf{b}}$} \\
\hline & wk 0 & wk 12 & wk $12-0$ & $P^{a}$ & wk 0 & wk 12 & wk $12-0$ & $P^{a}$ & \\
\hline Energy (kcal/d) & $2341.9 \pm 650.0$ & $\begin{array}{l}2440.4 \pm \\
758.4\end{array}$ & $98.5 \pm 601.3$ & 0.566 & $2234.4 \pm 950.3$ & $\begin{array}{c}2518.8 \pm \\
1095.4\end{array}$ & $284.5 \pm 1229.6$ & 0.223 & 0.593 \\
\hline Protein (g/d) & $95.6 \pm 22.7$ & $93.4 \pm 40.7$ & $-2.2 \pm 37.9$ & 0.498 & $90.6 \pm 45.7$ & $102.7 \pm 57.6$ & $12.1 \pm 67.3$ & 0.263 & 0.211 \\
\hline Protein (\%EN) & $16.89 \pm 2.23$ & $15.43 \pm 2.90$ & $-1.47 \pm 2.92$ & 0.027 & $16.27 \pm 3.10$ & $16.13 \pm 2.72$ & $-0.14 \pm 3.55$ & 0.592 & 0.198 \\
\hline Carbohydrates (g/d) & $237.0 \pm 59.3$ & $237.2 \pm 62.3$ & $0.2 \pm 58.1$ & 0.931 & $240.9 \pm 96.9$ & $241.1 \pm 86.0$ & $0.2 \pm 109.3$ & 0.808 & 0.771 \\
\hline Carbohydrates (\%EN) & $41.42 \pm 6.35$ & $40.53 \pm 7.14$ & $-0.89 \pm 5.83$ & 0.715 & $44.79 \pm 7.74$ & $40.27 \pm 7.65$ & $-4.51 \pm 7.53$ & 0.014 & 0.076 \\
\hline Fat $(\mathrm{g} / \mathrm{d})$ & $93.4 \pm 30.4$ & $106.6 \pm 37.6$ & $13.2 \pm 30.2$ & 0.068 & $89.1 \pm 47.0$ & $111.0 \pm 57.6$ & $21.9 \pm 60.5$ & 0.050 & 0.789 \\
\hline Fat (\%EN) & $36.4 \pm 5.5$ & $39.4 \pm 4.7$ & $3.03 \pm 6.16$ & 0.079 & $35.1 \pm 6.2$ & $39.1 \pm 5.8$ & $4.04 \pm 6.04$ & 0.006 & 0.423 \\
\hline Alcohol (g/d) & $21.3 \pm 35.4$ & $19.3 \pm 28.2$ & $-1.96 \pm 16.86$ & 0.476 & $11.8 \pm 20.2$ & $17.9 \pm 27.6$ & $6.03 \pm 24.84$ & 0.227 & 0.206 \\
\hline Alcohol (\%EN) & $5.33 \pm 7.61$ & $4.66 \pm 6.29$ & $-0.67 \pm 3.29$ & 0.590 & $3.86 \pm 6.05$ & $4.48 \pm 6.58$ & $0.62 \pm 5.65$ & 0.872 & 0.771 \\
\hline Cholesterol (mg/d) & $363.2 \pm 145.2$ & $393.6 \pm 213.7$ & $30.4 \pm 160.4$ & 0.543 & $332.4 \pm 189.0$ & $359.7 \pm 193.7$ & $27.2 \pm 259.2$ & 0.758 & 0.884 \\
\hline SAFA (g/d) & $34.6 \pm 13.9$ & $41.9 \pm 13.5$ & $7.3 \pm 11.7$ & 0.016 & $32.4 \pm 17.7$ & $40.2 \pm 18.8$ & $7.8 \pm 21.1$ & 0.072 & 0.942 \\
\hline$M C T(g / d)$ & $1.31 \pm 0.76$ & $7.74 \pm 0.56$ & $6.43 \pm 0.83$ & $<0.001$ & $1.08 \pm 0.64$ & $4.94 \pm 2.12$ & $3.86 \pm 2.29$ & $<0.001$ & $<0.001$ \\
\hline MUFA (g/d) & $34.0 \pm 12.3$ & $39.9 \pm 16.0$ & $5.9 \pm 13.5$ & 0.039 & $34.1 \pm 19.9$ & $45.5 \pm 26.5$ & $11.3 \pm 27.1$ & 0.010 & 0.627 \\
\hline PUFA (g/d) & $14.5 \pm 6.6$ & $16.8 \pm 5.7$ & $2.3 \pm 6.6$ & 0.033 & $14.7 \pm 7.7$ & $18.2 \pm 9.0$ & $3.4 \pm 9.5$ & 0.082 & 0.981 \\
\hline Oleic acid (g/d) & $30.1 \pm 11.2$ & $35.8 \pm 14.4$ & $5.7 \pm 12.5$ & 0.039 & $30.1 \pm 17.7$ & $40.7 \pm 24.5$ & $10.7 \pm 24.6$ & 0.011 & 0.610 \\
\hline LA (g/d) & $12.34 \pm 5.76$ & $13.79 \pm 4.98$ & $1.45 \pm 5.61$ & 0.073 & $12.91 \pm 6.88$ & $15.21 \pm 8.09$ & $2.30 \pm 8.30$ & 0.322 & 0.981 \\
\hline $\operatorname{ALA}(\mathrm{g} / \mathrm{d})$ & $1.35 \pm 0.82$ & $2.09 \pm 0.38$ & $0.74 \pm 0.78$ & 0.001 & $1.15 \pm 0.54$ & $2.03 \pm 0.66$ & $0.88 \pm 0.79$ & $<0.001$ & 0.409 \\
\hline LA/ALA & $9.60 \pm 1.87$ & $6.47 \pm 1.23$ & $-3.14 \pm 2.08$ & $<0.001$ & $11.37 \pm 3.70$ & $7.22 \pm 1.70$ & $-4.15 \pm 3.43$ & $<0.001$ & 0.395 \\
\hline$E P A(g / d)$ & $0.040 \pm 0.038$ & $0.187 \pm 0.035$ & $0.147 \pm 0.048$ & $<0.001$ & $0.035 \pm 0.039$ & $0.178 \pm 0.027$ & $0.143 \pm 0.049$ & $<0.001$ & 0.981 \\
\hline $\mathrm{DHA}(\mathrm{g} / \mathrm{d})$ & $0.075 \pm 0.085$ & $0.192 \pm 0.148$ & $0.117 \pm 0.167$ & 0.003 & $0.068 \pm 0.116$ & $0.152 \pm 0.071$ & $0.083 \pm 0.151$ & 0.001 & 0.752 \\
\hline $\mathrm{LA} /(\mathrm{ALA}+\mathrm{EPA}+\mathrm{DHA})$ & $8.72 \pm 1.63$ & $5.50 \pm 1.11$ & $-3.22 \pm 1,65$ & $<0.001$ & $10.56 \pm 3.67$ & $6.20 \pm 1.57$ & $-4.35 \pm 3.35$ & $<0.001$ & 0.296 \\
\hline Glucose (g/d) & $11.4 \pm 6.4$ & $13.7 \pm 6.1$ & $2.3 \pm 7.0$ & 0.106 & $14.7 \pm 7.4$ & $13.7 \pm 8.9$ & $-1.0 \pm 8.8$ & 0.661 & 0.174 \\
\hline Fructose $(\mathrm{g} / \mathrm{d})$ & $16.7 \pm 10.4$ & $17.7 \pm 8.8$ & $1.0 \pm 11.6$ & 0.532 & $20.2 \pm 9.3$ & $18.0 \pm 11.1$ & $-2.1 \pm 12.2$ & 0.527 & 0.296 \\
\hline Sorbitol (g/d) & $1.01 \pm 0.83$ & $1.25 \pm 1.12$ & $0.24 \pm 1.37$ & 0.498 & $1.01 \pm 0.58$ & $1.70 \pm 1.46$ & $0.69 \pm 1.49$ & 0.051 & 0.437 \\
\hline Retinol (RE, $\mu \mathrm{g} / \mathrm{d}$ ) & $\begin{array}{c}3093.4 \pm \\
6769.2\end{array}$ & $\begin{array}{c}1671.6 \pm \\
825.4\end{array}$ & $\begin{array}{l}-1421.8 \pm \\
6808.4\end{array}$ & 0.434 & $\begin{array}{c}2611.9 \pm \\
3613.9\end{array}$ & $1845.3 \pm 792.9$ & $\begin{array}{c}-766.5 \pm \\
3442.7\end{array}$ & 0.783 & 0.789 \\
\hline Vitamin $D(\mu \mathrm{g} / \mathrm{d})$ & $3.09 \pm 3.68$ & $5.54 \pm 4.76$ & $2.45 \pm 6.08$ & 0.010 & $2.48 \pm 3.58$ & $3.54 \pm 2.01$ & $1.06 \pm 4.36$ & 0.042 & 0.627 \\
\hline Vitamin E (mg/d) & $11.01 \pm 5.25$ & $14.45 \pm 4.25$ & $3.44 \pm 4.66$ & 0.004 & $12.10 \pm 5.65$ & $15.03 \pm 7.19$ & $2.92 \pm 7.60$ & 0.077 & 0.481 \\
\hline Vitamin B2 (mg/d) & $1.77 \pm 0.98$ & $2.18 \pm 0.65$ & $0.41 \pm 1.03$ & 0.008 & $1.59 \pm 0.72$ & $2.26 \pm 1.04$ & $0.67 \pm 1.24$ & 0.006 & 0.752 \\
\hline $\begin{array}{l}\text { Nicotinic acid (mg/ } \\
\text { d) }\end{array}$ & $22.5 \pm 8.8$ & $25.9 \pm 9.3$ & $3.5 \pm 12.2$ & 0.217 & $19.5 \pm 8.3$ & $29.3 \pm 15.0$ & $9.8 \pm 15.0$ & $<0.001$ & 0.159 \\
\hline Vitamin B6 (mg/d) & $2.11 \pm 0.80$ & $2.52 \pm 1.31$ & $0.41 \pm 1.17$ & 0.122 & $1.98 \pm 0.89$ & $2.75 \pm 1.31$ & $0.77 \pm 1.44$ & 0.002 & 0.296 \\
\hline Folic acid ( $\mu \mathrm{g} / \mathrm{d})$ & $250.4 \pm 157.3$ & $354.1 \pm 88.9$ & $103.7 \pm 119.1$ & 0.001 & $239.5 \pm 82.7$ & $371.5 \pm 139.4$ & $131.9 \pm 153.3$ & $<0.001$ & 0.716 \\
\hline Vitamin B12 ( $\mu \mathrm{g} / \mathrm{d})$ & $11.14 \pm 15.76$ & $10.54 \pm 4.74$ & $-0.61 \pm 16.15$ & 0.063 & $8.65 \pm 7.70$ & $10.83 \pm 5.19$ & $2.18 \pm 9.35$ & 0.050 & 0.369 \\
\hline Vitamin C (mg/d) & $92.8 \pm 89.2$ & $79.2 \pm 29.6$ & $-13.6 \pm 86.0$ & 0.848 & $88.9 \pm 40.6$ & $92.3 \pm 48.5$ & $3.4 \pm 64.1$ & 0.758 & 0.771 \\
\hline Sodium (g/d) & $3.14 \pm 1.23$ & $3.02 \pm 1.24$ & $-0.12 \pm 1.10$ & 0.715 & $3.39 \pm 1.87$ & $3.50 \pm 2.21$ & $0.11 \pm 2.54$ & 0.485 & 0.716 \\
\hline Chloride (g/d) & $4.74 \pm 1.71$ & $4.62 \pm 1.84$ & $-0.12 \pm 1.51$ & 0.741 & $5.03 \pm 2.65$ & $5.29 \pm 3.38$ & $0.26 \pm 3.86$ & 0.592 & 0.644 \\
\hline Salt (g/d) & $7.1 \pm 2.7$ & $6.9 \pm 2.8$ & $-0.2 \pm 2.4$ & 0.715 & $7.5 \pm 4.2$ & $7.8 \pm 5.1$ & $0.4 \pm 5.9$ & 0.509 & 0.789 \\
\hline Fiber (g/d) & $21.9 \pm 11.7$ & $22.2 \pm 7.2$ & $0.32 \pm 8.96$ & 0.434 & $24.1 \pm 10.5$ & $25.3 \pm 13.4$ & $1.16 \pm 14.47$ & 0.910 & 0.808 \\
\hline Purins (mg/d) & $203.9 \pm 67.0$ & $201.9 \pm 98.8$ & $-2.0 \pm 111.8$ & 0.821 & $200.7 \pm 98.6$ & $230.1 \pm 129.3$ & $27.0 \pm 145.5$ & 0.327 & 0.464 \\
\hline Uric acid (mg/d) & $613.4 \pm 201.4$ & $605.9 \pm 297.7$ & $-7.5 \pm 336.1$ & 0.794 & $586.3 \pm 279.8$ & $685.2 \pm 352.7$ & $98.9 \pm 397.4$ & 0.072 & 0.224 \\
\hline
\end{tabular}

\footnotetext{
1 data are presented as mean \pm SD

${ }^{a} p$ values for comparison between week 0 and 12 (Wilcoxon-Test)

b $\mathrm{p}$ values for comparison of group $\times$ time interactions (Mann-Whitney-U-Test)

2 abbreviations used: ALA, alpha-linolenic acid; DHA, docosahexaenoic acid; EN, energy; EPA, eicosapentaenoic acid; LA, linoleic acid; MCT, medium-chain triacylglycerols; MUFA, monounsaturated fatty acids; PUFA, polyunsaturated fatty acids; RE, retinol equivalent; SAFA, saturated fatty acids
}

observed in this study is in accordance with previous research evaluating the effect of MCT in overweight subjects [21-23]. The supplementation of $5 \mathrm{~g} / \mathrm{d}$ MCT in form of an oil for instance resulted in a significant decrease in WC compared to the long-chain triacylglycerols (LCT) control treatment $(-5.1 \pm 3.1 \mathrm{~cm}$ vs. $-3.3 \pm 1.9 \mathrm{~cm}, \mathrm{p}<$ $0.05)$ [21]. Tsuji et al. [23] reported a significant reduction in WC by $5.67 \pm 0.05 \mathrm{~cm}$ with $\mathrm{BMI} \geq 23 \mathrm{~kg} / \mathrm{m}^{2}$, however only in the area of subcutaneous fat, at a total daily MCT intake of $9.24 \mathrm{~g} / \mathrm{d}$. 
Since some evidence also pointed at a beneficial effect on satiety [24], it has been proposed that under free living conditions an increase in dietary MCT may result in less energy intake and contributes to weight management. However, the fact that we did not find any decrease in energy intake and no change in body weight suggests that the observed effect on WC may be rather ascribed to an effect on energy expenditure and thermogenesis than to an increase in satiety $[13,25,26]$.

Since long-chain PUFA are proposed to reduce TG, we hypothesized that the intervention may involve an improvement in dyslipidemia. However, in our study the consumption of the fat spreads did not result in any reductions in blood lipids. In contrast, we observed a significant rise in fasting TG in both groups after supplementation. It is suggested that fasting TG may have been affected by changes in dietary habits regarding the quantity and quality of fat intake. Fatty acid intake profile of patients revealed a rise in the intake from MUFA, especially oleic acid, in both groups. Table 4 shows that TG-lowering effect could be attributed only to MCT intake, whereas MUFA increased serum TG.

This rise in fat consumption may have counteracted any beneficial effects of the n-3-PUFA and may have caused the adverse effect on fasting TG instead. It further indicates that the spreadable fat has not fully been consumed as a replacement of other fat in the diet as intended, but rather in addition. In deed, several patients reported that the consumption of $30 \mathrm{~g} / \mathrm{d}$ of spread during the study was much higher than the amount of spread they usually consume which was reflected in their dietary protocols. However, observations in free-living conditions illustrated that users of plant sterol enriched margarines generally consumed less than the $20 \mathrm{~g} / \mathrm{d}$ recommended by the manufacturers. The average daily intake of phytosterol-enriched margarine ranged between 9 and 14 g [27], indicating that the serving size chosen in this study may not be consistent with habitual spread intake in Europe. Unpredictable effects such as changes in physical activity and nutritional behaviour over time which are difficult to control for may bias results even in carefully designed studies [28].

An additional effect on fasting TG may have been expected due to n-3-PUFA supplementation with the fat spreads. With combined daily intake of $240 \mathrm{mg}$ DHA + EPA and 870/900 mg ALA, the n-3-PUFA intake may have been too low to produce a triglyceride-lowering effect. The recommended daily intake of n-3-PUFA for the treatment of hypertriglyceridemia is 2 to $4 \mathrm{~g}$ [15].

The consumption of the fat spreads did not adversely affect fasting glucose, insulin, HOMA index, HbA1c, ASAT or ALAT. As GGT increased in MCT $\leq 7$ group, no general effect of MCT supplementation on GGT could be derived.

The observed reduction in urinary $\mathrm{pH}$ in the $\mathrm{MCT}>7$ group may be referred to the fact that MCT have a ketogenic character, as acetyl-CoA produced during medium-chain fatty acid oxidation is directed towards ketone body production [10]. Additionally, oral administration of MCT leads to urinary elimination of C6, C8 and C10 dicarboxylic acid [29,30].

Except for the MCT intake, no significant differences in macro- and micronutrient consumption were observed between both groups. Therefore, it is unlikely that the observed reduction in WC could be related to other nutritional factors than the MCT intake. It is suggested that the micronutrient intake with the fat spread was too low to achieve further beneficial cardio-metabolic effects.

In summary, a daily intake of at least $7 \mathrm{~g}$ MCT beneficially affects visceral fat mass, objectivised as WC, in overweight diabetic patients. As the daily intake of MCT through the normal diet is relatively small, a moderate enrichment of food with MCT may effectively contribute to achieve this required intake. The supply of MCT through enriched fat spreads may be adequate for individuals who are used to consume a high amount of spread on a daily basis. But, it may be reasonable to reassess the suitability of a fat spread as matrix for MCT. As dietary counselling for diabetic patients includes recommendations to reduce and modify the fat

Table 4 Serum fasting triglycerides depending on change of fatty acid intake ${ }^{1}$

\begin{tabular}{|c|c|c|c|c|c|c|c|}
\hline ANCOVA & $\begin{array}{l}\text { Oleic acid } \\
(\mathbf{g} / \mathrm{d})\end{array}$ & $\begin{array}{l}\text { MUFA* }^{*} \\
(\mathrm{~g} / \mathrm{d})\end{array}$ & $\begin{array}{l}\text { PUFA } \\
\text { (g/d) }\end{array}$ & $\begin{array}{l}\text { MCT } \\
\text { (g/d) }\end{array}$ & $\begin{array}{c}\text { SAFA*** } \\
(g / d)\end{array}$ & $\begin{array}{c}\text { Age } \\
\text { (years) }\end{array}$ & $\begin{array}{l}\text { Gender }^{2} \\
(1 \mathrm{w} / 2 \mathrm{~m})\end{array}$ \\
\hline PP & B: 0.789 & B: 2.046 & B: -1.710 & B: -17.950 & B: 0.324 & B: -0.050 & B: -72.396 \\
\hline$(n=43)$ & $\begin{array}{c}95 \% \mathrm{Cl} \\
(-9.84 ; 11.42)\end{array}$ & $\begin{array}{c}95 \% \mathrm{Cl} \\
(-44.8 ; 48.9) \\
\end{array}$ & $\begin{array}{c}95 \% \mathrm{Cl} \\
(-19.0 ; 15.6)\end{array}$ & $\begin{array}{c}95 \% \mathrm{Cl} \\
(-42.1 ; 6.2)\end{array}$ & $\begin{array}{c}95 \% \mathrm{Cl} \\
(-13.5 ; 14.2)\end{array}$ & $\begin{array}{c}95 \% \mathrm{Cl} \\
(-6.2 ; 6.1)\end{array}$ & $\begin{array}{c}95 \% \mathrm{Cl} \\
(-175.5 ; 30.7)\end{array}$ \\
\hline$p=0.471$ & $p=0.881$ & $p=0.930$ & $p=0.842$ & $p=0.141$ & $p=0.962$ & $p=0.987$ & $p=0.163$ \\
\hline
\end{tabular}

${ }^{*}$ without oleic acid

***without MCT

1 abbreviations used: MCT, medium-chain triacylglycerols; MUFA, monounsaturated fatty acids; PUFA, polyunsaturated fatty acids; SAFA, saturated fatty acids

2 gender is coded by 1 (women) and 2 (men) 
intake, alternative food matrices such as oil and milk products may be interesting alternatives in order to ensure a healthy overall diet. Since no significant differences in macro- and micronutrient consumption were observed between both groups, it is suggested that any beneficial effects of the n-3-PUFA and vitamins may have been counteracted by the increased intake of other fatty acids.

\section{Additional material}

Additional file 1: Trial profile ${ }^{1}$. The trial profile shows flow of the patients from screening to study completion. ' abbreviations used: ITT, intention to treat, MCT, medium-chain triacylglycerols; PP, per protocol

\section{Acknowledgements}

We would like to acknowledge Dr. Myslik and his team for their efforts on the conduct of the study.

\section{Author details}

'Medical Nutrition Science, Department of Urology, University of Bonn, Bonn, Germany. ${ }^{2}$ Bonn Education Association for Dietetics r. A., Cologne, Germany. ${ }^{3}$ Medicine and Service Ltd, Department of Biostatistics, Chemnitz, Germany. ${ }^{4}$ Department of Internal Medicine III, University Hospital, RWTH, Aachen, Germany.

\section{Authors' contributions}

CM designed research. RS was involved in project conception and overall research plan. CE analyzed dietary intake and conducted monitoring. NB performed statistical analysis. RS, CE and CM wrote paper. RS and CM have primary responsibility for final content. All authors read and approved the final manuscript.

\section{Competing interests}

This study was supported by a research grant from basis $\mathrm{GmbH}$, Woerthsee, Germany. The funder was neither involved in the study design,

interpretation of data, nor in the content of the final manuscript.

None of the authors has a conflict of interest for this manuscript others than that the study has been funded by basis $\mathrm{GmbH}$, Woerthsee, Germany. Prof. Dr. Dr. Metzner was a consultant for basis $\mathrm{GmbH}$, Woerthsee, Germany.

Received: 8 October 2010 Accepted: 8 April 2011 Published: 8 April 2011

\section{References}

1. Trayhurn P, Wood IS: Signalling role of adipose tissue: adipokines and inflammation in obesity. Biochem Soc Trans 2005, 33:1078-1081.

2. Kahn HS, Valdez R: Metabolic risks identified by the combination of enlarged waist and elevated triacylglycerol concentration. Am J Clin Nutr 2003, 78:928-934.

3. Sam S, Haffner S, Davidson MH, D'Agostino RB, Feinstein S, Kondos G, Perez A, Mazzone T: Hypertriglyceridemic waist phenotype predicts increased visceral fat in subjects with type 2 diabetes. Diabetes Care 2009, 32:1916-1620.

4. Blackburn P, Lemieux I, Alméras N, Bergeron J, Cote M, Tremblay A, Lamarche B, Despres JP: The hypertriglyceridemic waist phenotype versus the National Cholesterol Education Program-Adult Treatment Panel III and International Diabetes Federation clinical criteria to identify highrisk men with an altered cardiometabolic risk profile. Metabolism 2009, 58:1123-1130

5. Third report of the National Cholesterol Education Program (NCEP) Expert Panel on Detection, Evaluation, and Treatment of High Blood Cholesterol in Adults: Circulation 2002, 106:3143-3421.

6. Rogowski O, Shapira I, Steinvil A, Berliner S: Low-grade inflammation in individuals with the hypertriglyceridemic waist phenotype: another feature of the atherogenic dysmetabolism. Metabolism 2009, 58:661-667.
7. Rosolova H, Petrlova B, Simon J, Sifalda P, Sipova I: High-sensitivity Creactive protein and the hypertriglyceridemic waist in patients with type 2 diabetes and metabolic syndrome. Med Sci Monit 2008, 14:CR411-415.

8. Baynes JW, Thorpe SR: Role of oxidative stress in diabetic complications: a new perspective on an old paradigm. Diabetes 1999, 48:1-9.

9. Whiting PH, Kalansooriya A, Holbrook I, Haddad F, Jennings PE: The relationship between chronic glycaemic control and oxidative stress in type 2 diabetes mellitus. Br J Biomed Sci 2008, 65:71-74.

10. Bach AC, Ingenbleek $Y$, Frey A: The usefulness of dietary medium-chain triglycerides in body weight control: fact or fancy? J Lipid Res 1996, 37:708-726.

11. Beermann C, Jelinek J, Reinecker T, Hauenschild A, Boehm G, Kloer HU: Short term effects of dietary medium-chain fatty acids and $n-3$ longchain polyunsaturated fatty acids on the fat metabolism of healthy volunteers. Lipids Health Dis 2003, 2:1-10.

12. Johnson RC, Young SK, Cotter R, Lin L, Rowe WB: Medium-chaintriglyceride lipid emulsion: Metabolism and tissue distribution. Am J Clin Nutr 1990, 52:502-508.

13. Scalfi L, Coltorti A, Contaldo F: Postprandial thermogenesis in lean and obese subjects after meals supplemented with medium-chain and longchain triglycerides. Am J Clin Nutr 1991, 53:1130-1133.

14. Adkins $Y$, Kelly DS: Mechanisms underlying the cardioprotective effects of omega-3 polyunsaturated fatty acids. J Nutr Biochem 2010, 21:781-792.

15. Kris-Etherton PM, Harris WS, Appel L: Fish consumption, fish oil, omega-3 fatty acids, and cardiovascular disease. Circulation 2002, 106:2747-2757.

16. Zeman M, Zak A, Vecka M, Tvrzicka E, Pisarikova A, Stankova B: N-3 fatty acid supplementation decreases plasma homocysteine in diabetic dyslipidemia treated with statin-fibrate combination. J Nutr Biochem 2006, 17:379-384.

17. Hartweg J, Farmer AJ, Perera R, Holman RR, Neil HAW: Meta-analysis of the effects of $n-3$ polyunsaturated fatty acids on lipoproteins and other emerging lipid cardiovascular risk markers in patients with type 2 diabetes. Diabetologia 2007, 50:1593-1602

18. Zittermann A, Frisch S, Berthold HK, Goetting C, Kuhn J, Kleesiek K, Stehle P, Koertke $H$, Koerfer R: Vitamin D supplementation enhances beneficial effects of weight loss on cardiovascular risk markers. Am J Clin Nutr 2009, 89:1321-1327.

19. Shargorodsky M, Debby O, Matas Z, Zimlichman R: Effect of long-term treatment with antioxidants (vitamin C, vitamin E, coenzyme Q10 and selenium) on arterial compliance, humoral factors, and inflammatory markers in patients with multiple cardiovascular risk factors. Nutr Metab 2010, 7:55.

20. Levey AS, Coresh J, Greene T, Marsh J, Stevens LA, Kusek JW, Van Lente F: Expressing the modification of diet in renal disease study equation for estimating glomerular filtration rate with standardized serum creatinine values. Clin Chem 2007, 53:766-772.

21. Nosaka N, Maki H, Suzuki Y, Haruna H, Ohara A, Kasai M, Tsuji H, Toshiaki A, Okazaki M, Igarashi O, Kondo K: Effects of margarine containing mediumchain triacylglycerols on body fat reduction in humans. J Atheroscler Thromb 2003, 10:290-298.

22. Liu Y, Wang J, Zhang R, Zhang Y, Xu Q, Zhang J, Zhang Y, Zheng Z, Yu X, Jing H, Nosaka N, Kasai M, Aoyama T, Wu J, Xue C: A good response to oil with medium- and long-chain fatty acids in body fat and blood lipid profiles of male hypertriglyceridemic subjects. Asia Pac J Clin Nutr 2009, 18:351-358.

23. Tsuji H, Kasai M, Takeuchi H, Nakamura M, Okazaki M, Kondo K: Dietary medium-chain triacylglycerols suppress accumulation of body fat in a double-blind, controlled trial in healthy men and women. J Nutr 2001, 131:2853-2859.

24. Furuse M, Choi YH, Mabayo RT, Okumura Jl: Feeding behavior in rats fed diets containing medium chain triglyceride. Physiol Behav 1992, 52:815-817.

25. St-Onge MP, Ross R, Parsons WD, Jones PJH: Medium-chain triglycerides increase energy expenditure and decrease adiposity in overweight men. Obes Res 2003, 11:395-402.

26. Dulloo AG, Fathi M, Mensi N, Giardier L: Twenty-four-hour energy expenditure and urinary catecholamines of humans consuming low-tomoderate amounts of medium-chain triglycerides: a dose-response study in a human respiratory chamber. Eur J Clin Nutr 1996, 50:152-158.

27. Fransen $\mathrm{HP}$, de Jong $\mathrm{N}$, Wolfs $\mathrm{M}$, Verhagen $\mathrm{H}$, Verschuren WMM, Lütjohann D, von Bergmann K, Plat J, Mensink RP: Customary use of plant 
sterol and plant stanol enriched margarine is associated with changes in serum plant sterols and stanol concentrations in humans. J Nutr 2007, 137:1301-1306.

28. Zunft HJF, Lüder W, Harde A, Haber B, Graubaum HJ, Koebnick C, Grünwald J: Carob pulp preparation rich in insoluble fiber lowers total and LDL cholesterol in hypercholesterolemic patients. Eur J Nutr 2003, 42:235-242.

29. Mortensen PB: The possible antiketogenic and gluconeogenic effects of the beta-oxidation of fatty acids in rats. Biochim Biophys Acta 1980, 620:177-185.

30. Lima LAM, Gray OP, Losty H: Excretion of dicarboxylic acids following administration if medium chain triglycerides. J Parenter Enteral Nutr 1987, 11:600-601.

doi:10.1186/1743-7075-8-21

Cite this article as: Siener et al:: Effect of a fat spread enriched with medium-chain triacylglycerols and a special fatty acid-micronutrient combination on cardiometabolic risk factors in overweight patients with diabetes. Nutrition \& Metabolism 2011 8:21.

\section{Submit your next manuscript to BioMed Central} and take full advantage of:

- Convenient online submission

- Thorough peer review

- No space constraints or color figure charges

- Immediate publication on acceptance

- Inclusion in PubMed, CAS, Scopus and Google Scholar

- Research which is freely available for redistribution

Submit your manuscript at www.biomedcentral.com/submit
(Ciomed Central 